\title{
Actualización en el tratamiento de la encefalopatía hepática
}

\author{
R. García Martínez y J. Córdoba Cardona \\ Servicio Medicina Interna-Hepatología. Hospital Vall d'Hebron. Departamento de Medicina. Universitat Autònoma de \\ Barcelona. Centro de Investigación Biomédica en Red de Enfermedades Hepáticas y Digestivas (CIBEREHD). \\ Barcelona
}

García Martínez R, Córdoba Cardona J. Actualización en el tratamiento de la encefalopatía hepática. Rev Esp Enferm Dig 2008; 100: 637-644.

\section{INTRODUCCIÓN}

La encefalopatía hepática (EH) es un síndrome que incluye un espectro amplio de manifestaciones neurológicas y psiquiátricas, secundarias al efecto tóxico sobre el sistema nervioso de sustancias que en condiciones normales son eliminadas por el hígado (1). Aunque este principio fisiopatológico es sencillo, en él intervienen muchos elementos que afectan el metabolismo de las toxinas, la entrada de estas en el sistema nervioso central o su propio mecanismo lesivo. Además, se ha observado un efecto sinérgico entre varias de ellas sobre el estado mental. La realización de ensayos clínicos que demuestren la eficacia de los diversos tratamientos propuestos se ha visto dificultada por cómo estos múltiples factores pueden influir en la evolución de la EH (2). La mayoría de las recomendaciones terapéuticas se basan en conceptos derivados de estudios experimentales y de observaciones clínicas escasamente documentadas (3).

En los últimos años ha cobrado un interés renovado el papel del amoniaco en el desarrollo de la EH. Se conoce mejor su metabolismo en pacientes con cirrosis hepática (4) y se han propuesto nuevos mecanismos por los que se explica la disfunción cerebral (5). En la actualidad se están investigando una serie de moléculas que afectan al

Recibido: 23-07-08.

Aceptado: 29-07-08

Correspondencia: Juan Córdoba. Servicio de Medicina Interna-Hepatología. Hospital Vall d'Hebron. Paseo Vall d'Hebron 119. 08035 Barcelona. e-mail: jcordoba@vhebron.net metabolismo del amoniaco y que podrían tener un papel en la terapéutica.

\section{FISIOPATOLOGÍA}

La EH sucede como consecuencia de la exposición del cerebro a sustancias tóxicas por un fracaso en su eliminación hepática (6) (Fig. 1). Esto puede ocurrir por lesión del parénquima hepático o por la existencia de derivaciones portosistémicas. En ambos casos, múltiples sustancias del territorio venoso portal alcanzan la circulación

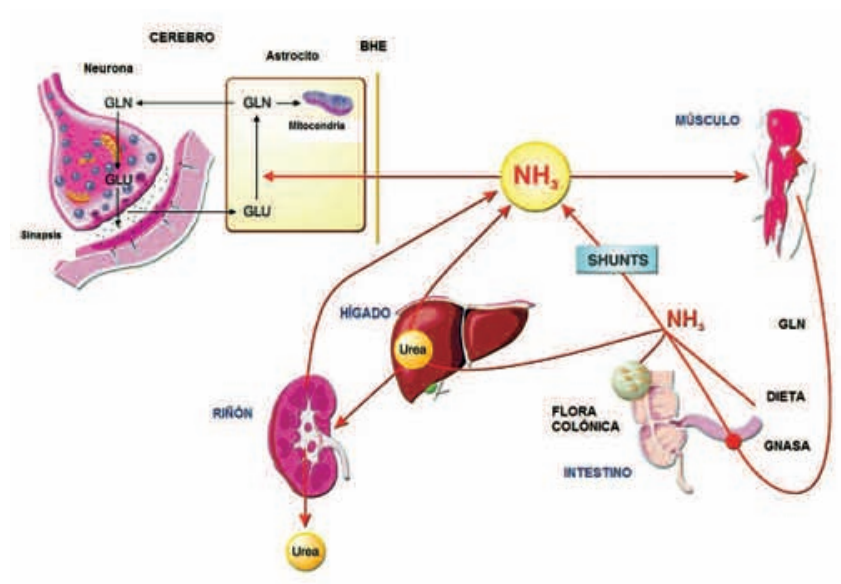

Fig. 1. Papel del amoniaco en la fisiopatología de la encefalopatía hepática (BHE: barrera hematoencefálica; Gln: glutamina; Glu: glutamato; Gnasa: glutaminasa; $\mathrm{NH}_{3}$ : amoniaco. 
sistémica. De todas ellas, se cree que el amoniaco es la más importante. El amoniaco procede mayoritariamente del intestino, en donde se genera tras el metabolismo de los productos nitrogenados de la dieta, la acción de la flora intestinal y el metabolismo de la glutamina por la glutaminasa intestinal. El $90 \%$ es metabolizado en el hígado dando lugar a la síntesis de urea que sufre posteriormente eliminación renal. En situación de cirrosis hepática, la contribución de otros órganos a la eliminación de amoniaco puede ser muy importante, destacando el papel del músculo esquelético, por su capacidad para la síntesis de glutamina (4). Una vez que el amoniaco atraviesa la barrera hematoencefálica es metabolizado a glutamina en el interior del astrocito, una reacción que consume mucha energía y podría conllevar estrés oxidativo y disfunción celular. La disfunción astrocitaria podría producir disfunción neuronal con alteración en la neurotransmisiónb (7). Los principales sistemas de neurotransmisión afectados son el mediado por glutamato (principal neurotransmisor excitador) y el mediado por GABA (principal neurotransmisión inhibidor) (8).

Además del amoniaco existen otros factores que participan en la EH, como el manganeso, una sustancia que se elimina por vía biliar y que se deposita en los ganglios de la base, donde ocasiona un incremento de señal T1 en la resonancia magnética. Otro factor que puede influir es la presencia de benzodiazepinas endógenas, que actúan sobre el receptor GABA. Recientemente ha cobrado especial importancia la inflamación (9). Se ha propuesto que la respuesta inflamatoria puede alterar la permeabilidad de la barrera hematoencefálica al efecto de diferentes toxinas o exacerbar las alteraciones cerebrales y ser responsable del desarrollo de episodios de encefalopatía hepática aguda en pacientes previamente compensados (10).

\section{CONSIDERACIONES CLÍNICAS}

La EH engloba un amplio espectro de manifestaciones clínicas, de gravedad muy variable, desde formas asintomáticas y sólo detectadas en test neuropsicológicos hasta el coma hepático (1). La mayoría de los pacientes presentan una cirrosis hepática, pero la EH también puede ser secundaria a trastornos de la vascularización hepática sin lesión del parénquima. En función de las manifestaciones neurológicas se pueden distinguir cuatro formas.

1. Encefalopatía hepática aguda. Es la forma más típica. Se caracteriza por una disminución brusca del nivel de conciencia, habitualmente asociada a un factor precipitante. El tratamiento del episodio de $\mathrm{EH}$ se dirige fundamentalmente a corregir el factor precipitante. Si se corrige sin que se altere la función hepática, el paciente vuelve a la situación previa al episodio de $\mathrm{EH}$.

2. Encefalopatía hepática crónica. Consiste en la existencia de episodios recurrentes de síndrome confusional o síntomas neurológicos persistentes (ataxia, disartria, temblor). Se ve en pacientes con cirrosis hepática que presentan grandes colaterales porto-sistémicas, bien sean espontáneas, quirúrgicas o secundarias a derivación portosistémica percutánea intrahepática (DPPI). Suele revertir con la oclusión de la colateral (11). En algunos pacientes las manifestaciones son graves y consisten en demencia o mielopatía.

3. Encefalopatía hepática mínima. Corresponde a un trastorno cognitivo que se pone de manifiesto en pruebas neuropsicológicas o neurofisiológicas puesto que los pacientes muestran un aspecto normal en la exploración neurológica estándar (12). Es muy frecuente y afecta a una gran proporción de pacientes con cirrosis hepática. Se relaciona con el grado de insuficiencia hepática, pero también con la edad y la presencia de derivaciones portosistémicas. A pesar de su carácter subclínico se ha demostrado que empeora la calidad de vida (13), por lo que se ha propuesto explorar la presencia de encefalopatía hepática mínima mediante la realización de pruebas psicométricas a todos los pacientes con cirrosis hepática.

4. Encefalopatía asociada a colaterales porto-sistémicas sin enfermedad hepática. Es un síndrome infrecuente asociado a colaterales congénitas (14), trombosis portal (15) o derivaciones quirúrgicas, en ausencia de enfermedad hepática parenquimatosa. Si tienen repercusión clínica suele ser en forma de síndrome confusional agudo o alteraciones neurológicas persistentes.

\section{TRATAMIENTO DE LA ENCEFALOPATÍA HEPÁTICA}

El tratamiento de la EH se dirige a una serie de dianas que determinan la concentración plasmática de las sustancias que tienen un efecto tóxico en el sistema nervioso central. La mayoría de tratamientos se han diseñado para disminuir la concentración plasmática de amoniaco (16). Además de los fármacos que se emplean para la $\mathrm{EH}$, se deben establecer una serie de medidas generales (nutrición, hidratación, aspiración de secreciones, protección de la vía aérea, tratamiento de infecciones, etc.), cuyo beneficio proviene de la experiencia clínica. En muchos casos no existe una explicación de los mecanismos de acción de estas medidas o son independientes de la acción sobre los potenciales tóxicos. Por ejemplo, se conoce bien que la corrección de la infección mejora la encefalopatía hepática precipitada por esta, pero se desconoce si es porque afecta la concentración de amoniaco o si afecta a mediadores de la inflamación.

Los estudios que avalan las recomendaciones terapéuticas son escasos y en gran número adolecen de un buen diseño. Como se ha comentado previamente, el término EH engloba condiciones clínicas muy diferentes, con pronósticos muy variables. Se ha asumido que si se demuestra la eficacia de un tratamiento para una situación clínica (p. ej. EH aguda sin factor precipitante) será válida para otra situación (p. ej. EH mínima), pero ello puede ser falso. Un número importante de estudios ha compara- 
do el tratamiento que se investiga frente a disacáridos no absorbibles, porque han considerado que no era ético compararlo a placebo. Sin embargo, un análisis crítico de los estudios de disacáridos no absorbibles ha llegado a la conclusión que no se ha demostrado su superioridad frente a placebo (3). Por ello actualmente se considera que los estudios con nuevos fármacos deben incluir un brazo con placebo.

Si bien el diagnóstico de la encefalopatía hepática no suele plantear grandes dificultades, su sintomatología es común a un gran número de enfermedades neurológicas con las que se puede confundir. Un número importante de enfermos puede presentar otras formas de lesión neurológica debidas al alcohol o malnutrición, que puede ser difícil de reconocer. Un aspecto controvertido es qué medidas generales deben ser consideradas las estándares. Si bien se considera que el tratamiento de los factores precipitantes es muy importante, lo estudios no suelen presentar el algoritmo empleado para el reconocimiento de los factores precipitantes y cómo se corrigieron.

\section{Disminución en la producción de amoniaco intestinal}

La experiencia clínica relaciona el desarrollo de encefalopatía con el contenido de compuestos nitrogenados en el tubo digestivo. Es por ello que los primeros tratamientos se dirigieron a la reducción de la producción de amonio intestinal. Con este propósito se desarrollaron fármacos que disminuyen la producción de amoniaco por la flora intestinal (disacáridos no absorbibles, antibióticos) y se recomendó disminuir la ingesta de proteínas en la dieta. Estas recomendaciones han constituido la base del tratamiento desde hace décadas. Sin embargo, han sido puestas en entredicho ante la constatación que los datos de los estudios que comparaban disacáridos no absorbibles frente a placebo no demostraban la superioridad de estos y que la reducción drástica de proteínas en la dieta no resultaba en una mejoría de la encefalopatía hepática aguda (17).

\section{Disacáridos no absorbibles}

Los disacáridos no absorbibles son moléculas constituidas por dos monosacáridos que no pueden ser absorbidas en el intestino delgado, debido a la ausencia de disacaridasas en la mucosa y llegan sin digerir al colon donde son metabolizadas por la flora intestinal sacarolítica. Existen dos preparados: lactulosa (ß-galactosido-fructosa) y lactitol ( $($-galactosido-sorbitol). Ambos tienen el mismo mecanismo de acción, que consiste en aumentar la incorporación de productos nitrogenados presentes en la luz intestinal a la flora bacteriana. Su acción se asocia a la consecución de un $\mathrm{pH}$ ácido, que se consigue por la generación durante su metabolismo bacteriano de ácidos grasos de cadena corta. Esta acidez desplaza el equilibrio del amoniaco hacia una mayor proporción de ión amonio, al cual resultan impermeables las mucosas. Ello tiene un efecto de atrapamiento que disminuye el paso de amoniaco al compartimento intravascular. Combinado con un efecto catártico, los disacáridos no absorbibles favorecen la expulsión de la flora que ha incorporado productos nitrogenados y se produce un efecto de salida del amoniaco (18). Además, al promover la flora sacarolítica sobre la proteolítica disminuye la proporción de bacterias que contienen ureasa y la formación de amoniaco a partir de urea presente en luz colónica. La consecuencia neta de la administración de disacáridos no absorbibles es una disminución de la concentración de amoniaco plasmático (19). Este mecanismo de acción es compatible con el empleo de fármacos que actúan a otro nivel, como podrían ser los que inhibiesen la acción de la glutaminasa intestinal o favoreciesen la eliminación de amoniaco en forma de urea por la orina. La administración de antibióticos puede eliminar la flora intestinal, que es precisa para que se metabolicen los disacáridos, lo que podría explicar los efectos negativos de la combinación con neomicina observados en un estudio (20). Una manera de comprobar si tiene lugar la metabolización de los disacáridos y por tanto si estos resultan eficaces cuando se combinan con antibióticos, es determinar el $\mathrm{pH}$ de las heces, que debe ser próximo a 5. En la práctica no es necesaria la monitorización del $\mathrm{pH}$ cuando se administran solos, debido a que este $\mathrm{pH}$ se asocia al incremento en el número de deposiciones.

La eficacia clínica de lactulosa y de lactitol es la misma, sin embargo lactitol parece ser tolerado mejor, debido a un mejor sabor y a que produce menos meteorismo (21-23). Existen pocos estudios que comparen los disacáridos no absorbibles con placebo. El análisis combinado muestra que mejora la evolución de la EH. Sin embargo, los resultados no fueron homogéneos y el análisis de los ensayos clínicos de mayor calidad que compararon disacáridos con placebo no encontraron diferencias a favor del grupo de tratamiento. Aunque se ha cuestionado el empleo de disacáridos no absorbibles, cabe destacar que el número de pacientes incluido en estos estudios es muy pequeño (44 pacientes) y que fueron realizados hace más de treinta años. Diversos autores han mostrado su apoyo al uso de disacáridos no absorbibles, que se basa en una larga experiencia de uso y en la demostración experimental de sus efectos sobre el amoniaco (24). Estudios recientes realizados en la India, que son posteriores al metaanálisis, e incluyen un número importante de pacientes han mostrado efectos beneficiosos de lactulosa en la encefalopatía hepática mínima $(25,26)$.

Los disacáridos no absorbibles son fármacos con los que existe una gran experiencia clínica, son bien tolerados y seguros, motivos por los que a pesar de una escasa documentación de su eficacia, siguen considerándose el tratamiento de elección. Se pueden administrar por vía oral (40-90 g/día) en 2 ó 3 tomas al día. La dosis se ajusta hasta conseguir 2-3 deposiciones blandas al día. También 
pueden usarse en enemas, mezclados con agua. Con el tiempo puede ser necesario aumentar la dosis para mantener el mismo efecto. Pueden producir diarrea por su efecto catártico. Un error, que no es infrecuente en pacientes hospitalizados, es incrementar la dosis de forma excesiva en pacientes que no mejoran, lo cual puede provocar de forma paradójica un empeoramiento de la $\mathrm{EH}$, debido a deshidratación.

\section{Antibióticos}

La introducción de antibióticos en el tratamiento de la EH se basa en los efectos de estos sobre la flora intestinal. Inicialmente se planteó que la principal fuente de amoniaco fuese la producción bacteriana, pero esto se rebatió con la demostración de que la ausencia de flora intestinal no prevenía el incremento de amoniaco causado por la insuficiencia hepática (27). Actualmente se considera que existe una contribución de la flora intestinal en determinar la concentración de amoniaco plasmático, pero que esta es menos importante de lo que se había creído. Se ha propuesto que los efectos beneficiosos de los antibióticos no se deban a que eliminen la flora intestinal que produce amoniaco. La neomicina reduce la producción intestinal de amoniaco inhibiendo la actividad de la glutaminasa (28), una enzima cuya actividad está incrementada en los pacientes con cirrosis hepática, lo que podría explicar en parte el incremento de amoniaco plasmático y la aparición de EH en estos pacientes (28). Otro posible mecanismo de acción de los antibióticos en la EH podría ser disminuyendo la traslocación bacteriana, la presencia de productos bacterianos en plasma y la respuesta inflamatoria a estos (29). Diversos datos sugieren que los mediadores de la inflamación pueden afectar la función mental y potenciar los efectos del amoniaco en el sistema nervioso central (30).

El primer antibiótico que se introdujo en la terapéutica fue la neomicina oral (31), un aminoglucósido que al no ser absorbible obviaba la toxicidad renal o auditiva de este grupo de fármacos. Se realizaron diversos estudios que avalaron su eficacia (32). Estos resultados han sido reafirmados por un meta-análisis, que muestra una leve superioridad frente a los disacáridos no absorbibles (3). Además de la neomicina se han propuesto otros antibióticos que podrían ser mas potentes, como el metronidazol o la vancomicina (33). Si bien la experiencia de uso es más limitada (34), existe una preocupación por su potencial efecto tóxico. Estas prevenciones se extienden a la neomicina (35), en especial con su uso prolongado. Por ello la mayoría de autores recomiendan evitar estos compuestos por periodos superiores a seis meses (36).

Un antibiótico que tiene buenas expectativas es la rifaximina (37), un forma no absorbible de la rifamicina. Este fármaco tiene actividad in vitro frente a organismos gram-negativos y anaerobios, y ocasiona una disminución importante de la flora bacteriana pocos días después de iniciado su uso. Su tolerancia es excelente y se ha empleado con éxito en diversos trastornos intestinales, como la diarrea del viajero o la diverticulitis aguda. Al igual que con la neomicina, se ha documentado bien su eficacia en el tratamiento de la EH aguda (38). Se está estudiando su empleo en la prevención de la recidiva y su seguridad a largo plazo. Hasta la actualidad no se ha identificado el desarrollo de mutantes resistentes, como ocurre con la rifampicina, un fármaco de la misma familia.

\section{Medidas dietéticas}

Se ha relacionado la EH con el aporte de proteínas en la dieta y la consiguiente producción de amoniaco. De ahí que se haya recomendado la restricción proteica en pacientes con EH. Sin embargo, el hecho que la ingesta de grandes cantidades de proteínas induzca $\mathrm{EH}$, no implica que la restricción proteica mejore su curso. El único ensayo clínico doble-ciego que ha estudiado dos dietas con diferente cantidad de proteínas en la EH aguda observó que no existía una mejoría de la evolución de la EH en el grupo con menor cantidad de proteínas y en cambio presentaban un mayor catabolismo proteico (39).

El metabolismo nitrogenado es complejo y en los últimos años se ha identificado un papel importante de diferentes tejidos, como el músculo esquelético y el riñón $(4,40)$. En este sentido es importante señalar que la cirrosis hepática se acompaña de malnutrición energético-proteica. Debido a que el músculo esquelético contribuye a la eliminación de amoniaco con la síntesis de glutamina, la ganancia de masa muscular podría contribuir a mejorar la encefalopatía hepática. Una dieta hipoproteica, además de haberse asociado a una peor supervivencia (41), puede disminuir la masa muscular y empeorar la $\mathrm{EH}$.

Asimismo estudios experimentales muestran que la eliminación completa de proteínas en la dieta conducen a un incremento del amoniaco plasmático, debido a que se disminuye la actividad de la carbamil-fosfato-sintetasa, un enzima del ciclo de la urea (42).

La recomendación actual es que los pacientes con cirrosis hepática y $\mathrm{EH}$ reciban una dieta normoproteica $(0,8-1,0 \mathrm{~g} / \mathrm{kg} / \mathrm{d})$ (43). En los casos con grados importantes de malnutrición se puede conseguir un balance nitrogenado positivo con el incremento de la cantidad de proteínas en la dieta, pero ello debe realizarse con precaución porque las dosis elevadas pueden inducir EH (44).

En cuanto a las características de la dieta (45), se ha observado una superioridad de dietas con proteínas de origen vegetal respecto a las de origen animal. Es posible que este beneficio se deba a que las dietas con proteínas animales son deficitarias en isoleucina y que la dieta vegetal aporte fibra. Por otra parte, los preparados nutricionales ricos en aminoácido de cadena ramificada pueden ser útiles en pacientes con EH crónica. Sus efectos anabólicos y energéticos a nivel muscular podrían favorecer una disminución del amoniaco y un mejor curso clínico (46). 
Durante el episodio de EH aguda suele ser necesario evitar la vía oral debido a la disminución de la conciencia. Al principio se emplean suplementos parenterales de glucosa. Al cabo de tres o cuatro días se reinicia la dieta oral sin restricciones en la cantidad de proteínas o se instaura nutrición artificial, preferiblemente por vía enteral. Es importante evitar el ayuno prolongado con ingestas múltiples de cantidades pequeñas (4-6 veces/día), para evitar la neoglucogénesis. Los datos actuales no demuestran ningún beneficio de la infusión de aminoácidos de cadena ramificada en la EH aguda (47). Además debe tratarse el déficit de vitaminas y minerales. En pacientes con cirrosis alcohólica es aconsejable aportar tiamina y en algunos casos zinc.

\section{Corrección de los factores precipitantes}

En la mayoría de los casos de EH aguda y en algunos casos de EH crónica recurrente subyace un factor precipitante. Inicialmente puede ser difícil de objetivar por lo que se ha de realizar una búsqueda sistemática de los mismos (Tabla I). Se han de buscar procesos infecciosos intercurrentes, incluida la peritonitis bacteriana espontánea y la bacteriemia espontánea del paciente cirrótico y ante la mínima sospecha se ha de iniciar tratamiento antibiótico empírico. Asimismo se han de buscar alteraciones hidroelectrolíticas y del equilibrio ácido base. Estos son muy importantes puesto que la alcalosis favorece el paso del amoniaco a través de la barrera hematoencefálica y la hipopotasemia aumenta la producción renal de amoniaco. Asimismo, la deshidratación dificulta la excreción renal de productos nitrogenados por lo que debe ser tratada y prevenirse. La hemorragia digestiva, el estreñimiento y la dieta hiperproteica son otros factores que cabe identificar y tratar.

\section{Flumazenilo}

El flumazenilo es un antagonista del receptor de las benzodiazepinas en el sistema de neurotransmisión mediado por GABA. Su empleo en la EH proviene de la observación de que algunos pacientes presentan en plasma sustancias que reaccionan con este receptor (48). Estas sustancias pueden corresponder al empleo subrepticio de benzodiazepinas farmacológicas, a las que los pacientes muestran mayor susceptibilidad o benzodiazepinas de origen endógeno, cuya naturaleza no está aclarada todavía (49). Se han realizado diversos estudios que muestran mejoría inmediata de la $\mathrm{EH}$ en algunos pacientes. Un metaanálisis que incluye 6 estudios y 641 pacientes demostró una mejoría en el grupo de tratamiento pero el efecto fue transitorio y en un subgrupo de pacientes con buen pronóstico (50). No se pudo demostrar beneficio en la mejoría global ni en la supervivencia. Actualmente se reserva el uso de flumazenilo para los pacientes con EH relacionada con el consumo de benzodiazepinas y para enfermos en coma, en los que podría evitar la necesidad de proteger la vía aérea. Se ensaya una dosis y en caso de respuesta, que ha de ser inmediata, se puede repetir o administrar dosis de mantenimiento.

\section{Oclusión de colaterales portosistémicas}

La relación entre colaterales porto-sistémicas y el desarrollo de EH es bien conocido. La oclusión de las mismas podría ser una buena opción terapéutica en pacientes adecuadamente seleccionados (51). Los mejores candidatos son pacientes con EH recurrente, con función hepática relativamente preservada y bajo riesgo de hemorragia por hipertensión portal (52). La técnica de elección en pa-

Tabla I. Factores precipitantes

\begin{tabular}{|c|c|c|}
\hline Factor precipitante & Diagnóstico & Tratamiento \\
\hline Hemorragia gastrointestinal & $\begin{array}{l}\text { Examen del contenido gástrico y rectal, } \\
\text { endoscopia digestiva }\end{array}$ & $\begin{array}{l}\text { Tratamiento de la hemorragia } \\
\text { Enema de limpieza }\end{array}$ \\
\hline Estreñimiento & Historia clínica & Enema de limpieza \\
\hline Dieta hiperproteica & Historia clínica & Enema de limpieza \\
\hline Psicofármaco & $\begin{array}{l}\text { Historia clínica, determinación del fármaco } \\
\text { en plasma u orina }\end{array}$ & Antídotos (flumazenilo, naloxona) \\
\hline Insuficiencia renal & Determinación de creatinina en plasma, ecografía renal & $\begin{array}{l}\text { Suspender diuréticos y fármacos nefrotóxicos } \\
\text { Tratamiento específico de la causa }\end{array}$ \\
\hline Alteración de electrolitos & Determinación de electrolitos plasmáticos & Suspender diuréticos, corregir alteración de electrolitos \\
\hline Infección & $\begin{array}{l}\text { Cultivos de sangre u otras muestras corporales; } \\
\text { paracentesis (ascitis) o toracocentesis (derrame pleural) }\end{array}$ & $\begin{array}{l}\text { Ante la sospecha iniciar antibióticos de amplio } \\
\text { espectro en espera de los resultados de los cultivos }\end{array}$ \\
\hline Lesión hepática sobreañadida & $\begin{array}{l}\text { Historia clínica, enzimas hepáticos, ecografía hepática, } \\
\text { biopsia hepática }\end{array}$ & $\begin{array}{l}\text { Algunos casos pueden beneficiarse de medidas } \\
\text { específicas }\end{array}$ \\
\hline
\end{tabular}


cientes con cirrosis es por radiología intervencionista, por ser más segura y presentar menos complicaciones que la cirugía. En el caso de las colaterales portosistémicas congénitas debe descartarse previamente la presencia de hipoplasia severa de la vena porta puesto que la oclusión de la colateral podría desencadenar hemorragia digestiva por hipertensión portal.

La DPPI es un recurso terapéutico en pacientes con ascitis refractaria o hemorragia digestiva por varices. Uno de los principales efectos adversos de esta técnica es la aparición de encefalopatía. Suele aparecer en los primeros meses tras el procedimiento en una tercera parte de los pacientes. Los factores de riesgo asociados a la aparición de la misma son: episodios previos de encefalopatía, edad avanzada ( $>65$ años) y una mala función hepática previa (Child-Pugh C). Se ha propuesto el uso profiláctico de disacáridos no absorbibles o antibióticos sin embargo no se pudo demostrar que su uso disminuya la incidencia de encefalopatía en estos pacientes (53). Por ello no se recomienda el uso de tratamiento preventivo. En casos de episodios de encefalopatía hepática grave tras la colocación de DPPI se debe plantear la colocación de una prótesis de menor calibre valorando el riesgo de hemorragia por hipertensión portal secundario a dicha manipulación (54).

\section{Terapias futuras}

\section{Moduladores del metabolismo del amoniaco}

Existe una serie de fármacos que están siendo investigados para reducir el amoniaco plasmático a través de diferentes mecanismos. Algunos de ellos han sido empleados con éxito en pacientes con trastornos del ciclo de la urea (55), otros se han desarrollado específicamente para el tratamiento de la $\mathrm{EH}$ en pacientes con cirrosis hepática (56).

L-Ornitina L-aspartato (LOLA) es un fármaco con el que existe experiencia en Asia y Latinoamérica (57), que está disponible en Alemania y Austria, pero no se ha comercializado en España. Es una sal que se emplea por vía endovenosa, constituida por dos aminoácidos naturales, que constituyen un sustrato en el catabolismo del amoniaco. Inducen un aumento del metabolismo hepático y muscular del amoniaco a urea y glutamina. Se ha promovido como factor nutricional. Precisaría nuevos estudios que confirmasen su eficacia en la EH.

L-Ornitina fenilacetato es un nuevo fármaco con un doble mecanismo de acción (58). L-Ornitina estimula la síntesis de glutamina en el músculo a expensas de amoniaco. El fenilacetato excreta la glutamina dependiente de la L-Ornitina por la orina. Es un fármaco en fase experimental con resultados positivos en modelos animales.

Los probióticos se han empleado en estudios piloto $(59,60)$ en los que han demostrado una mejoría de la $\mathrm{EH}$.
El mecanismo de acción parece que relaciona la disminución de amoniaco plasmática con la colonización de bacterias no productoras de ureasa ácido-resistentes. Sin embargo su uso no se ha generalizado y su papel en la práctica clínica habitual está por definir.

\section{Terapias de detoxificación}

La diálisis con albúmina (MARS) se ha propuesto como un sistema sustitutivo de detoxificación en situaciones de insuficiencia hepática aguda grave y en situaciones de hepatopatía crónica con un deterioro agudo grave. Los trabajos publicados tienen muchas limitaciones de diseño (número muy reducido de pacientes, estudios no controlados) sin embargo esta técnica parece ofrecer resultados positivos en cuanto a que mejora la situación hemodinámica, la sintomatología derivada de la hiperbilirrubinemia y la EH. Estos resultados parecen alentar su uso en pacientes viables y como terapia de soporte en pacientes pendientes de trasplante hepático. Se ha realizado un ensayo clínico en pacientes con EH en situación de insuficiencia hepática grave, en el que ha mostrado que es superior al tratamiento estándar (61). La mejoría sobre la EH parece clara, pero al no tener efectos sobre supervivencia queda por definir su papel en la terapéutica.

\section{AGRADECIMIENTOS}

La Dra. Rita García Martínez disfruta de una Beca post-Formación Sanitaria Especializada del Instituto de Salud Carlos III. CIBEREHD está financiado por el Instituto de Salud Carlos III.

\section{BIBLIOGRAFÍA}

1. Ferenci P, Lockwood A, Mullen K, Tarter R, Weissenborn K, Blei AT. Hepatic encephalopathy--definition, nomenclature, diagnosis, and quantification: final report of the working party at the 11th World Congresses of Gastroenterology. Vienna, 1998. Hepatology 2002; 35 (3): 716-21.

2. Córdoba J, Mínguez B, Vergara M. Treatment of hepatic encephalopathy. Lancet 2005; 365 (9468): 1384-5.

3. Als-Nielsen B, Gluud LL, Gluud C. Non-absorbable disaccharides for hepatic encephalopathy: systematic review of randomised trials. Br Med J 2004; 328: 1046.

4. Olde Damink SW, Jalan R, Redhead DN, Hayes PC, Deutz NE, Soeters PB. Interorgan ammonia and amino acid metabolism in metabolically stable patients with cirrhosis and a TIPSS. Hepatology 2002; 36 (5): 1163-71.

5. Balata S, Damink SWMO, Ferguson K, et al. Induced hyperammonemia alters neuropsychology, brain MR spectroscopy and magnetization transfer in cirrhosis. Hepatology 2003; 37 (4): 931-9.

6. Córdoba J, Mínguez B. Hepatic encephalopathy. Semin Liver Dis 2008; 28 (1): 70-80. 
7. Haussinger D, Kircheis G, Fischer R, Schliess F, vom Dahl S. Hepatic encephalopathy in chronic liver disease: a clinical manifestation of astrocyte swelling and low-grade cerebral edema? J Hepatol 2000; 32 (6): 1035-8.

8. Butterworth RF. Pathogenesis of hepatic encephalopathy: new insights from neuroimaging and molecular studies. J Hepatol 2003; 39 (2): 278-85.

9. Blei AT. Infection, inflammation and hepatic encephalopathy, synergism redefined. J Hepatol 2004; 40 (2): 327-30.

10. Wright G, Davies NA, Shawcross DL, et al. Endotoxemia produces coma and brain swelling in bile duct ligated rats. Hepatology 2007; 45 (6): 1517-26.

11. Ishikawa Y, Yoshida H, Mamada Y, et al. Surgical disconnection of patent paraumbilical vein in refractory hepatic encephalopathy. J Nippon Med Sch 2008; 75 (3): 152-6.

12. Ortiz M, Jacas C, Cordoba J. Minimal hepatic encephalopathy: diagnosis, clinical significance and recommendations. J Hepatol 2005; 42 (Supl. 1): S45-S53.

13. Arguedas MR, DeLawrence TG, McGuire BM. Influence of hepatic encephalopathy on health-related quality of life in patients with cirrhosis. Dig Dis Sci 2003; 48 (8): 1622-6.

14. Ortiz M, Cordoba J, Alonso J, et al. Oral glutamine challenge and magnetic resonance spectroscopy in three patients with congenital portosystemic shunts. J Hepatol 2004; 40 (3): 552-7.

15. Minguez B, Garcia-Pagan JC, Bosch J, et al. Noncirrhotic portal vein thrombosis exhibits neuropsychological and MR changes consistent with minimal hepatic encephalopathy. Hepatology 2006; 43 (4): $707-$ 14.

16. Blei AT, Cordoba J. Hepatic encephalopathy. Practice guidelines of the American College of Gastroenterology. Am J Gastroenterol 2001; 96 (7): 1968-76.

17. Shawcross DL, Jalan R. Dispelling myths in the treatment of hepatic encephalopathy. Lancet 2005; 365: 431-3.

18. Weber Jr FL, Fresard KM. Comparative effects of lactulose and magnesium sulfate on urea metabolism and nitrogen excretion in cirrhotic subjects. Gastroenterology 1981; 80: 994-8.

19. Weber FL, Jr., Fresard KM, Lally BR. Effects of lactulose and neomycin on urea metabolism in cirrhotic subjects. Gastroenterology 1982; 82: 213-7.

20. Blanc P, Daures JP, Liautard J, et al. Association lactulose-neomycine versus placebo dans le traitement de l'encephalopathie hepatique aigue. Resultats d'un essai controle randomise. Gastroenterol Clin Biol 1994; 18 (12): 1063-8.

21. Blanc P, Daures JP, Rouillon JM, et al. Lactitol or lactulose in the treatment of chronic hepatic encephalopathy: results of a meta-analysis. Hepatology 1992; 15 (2): 222-8.

22. Camma C, Fiorello F, Tine F, Marchesini G, Fabbri A, Pagliaro L. Lactitol in treatment of chronic hepatic encephalopathy. A metaanalysis. Dig Dis Sci 1993; 38 (5): 916-22.

23. Morgan MY, Hawley KE. Lactitol vs. lactulose in the treatment of acute hepatic encephalopathy in cirrhotic patients: a double-blind, randomized trial. Hepatology 1987; 7 (6): 1278-84.

24. Blei AT. Treatment of hepatic encephalopathy. Lancet 2005; 365 (9468): 1383-4.

25. Prasad S, Dhiman RK, Duseja A, Chawla YK, Sharma A, Agarwal R. Lactulose improves cognitive functions and health-related quality of life in patients with cirrhosis who have minimal hepatic encephalopathy. Hepatology 2007; 45 (3): 549-59.

26. Sharma P, Sharma BC, Puri V, Sarin SK. An open-label randomized controlled trial of lactulose and probiotics in the treatment of minimal hepatic encephalopathy. Eur J Gastroenterol Hepatol 2008; 20 (6): 506-11.

27. Schalm SW, van der Mey T. Hyperammonemic coma after hepatectomy in germ-free rats. Gastroenterology 1979; 77 (2): 231-4.

28. Romero-Gomez M, Ramos-Guerrero R, Grande L et al. Intestinal glutaminase activity is increased in liver cirrhosis and correlates with minimal hepatic encephalopathy. J Hepatol 2004; 41 (1): 49-54.

29. Frances R, Zapater P, Gonzalez-Navajas JM, et al. Bacterial DNA in patients with cirrhosis and noninfected ascites mimics the soluble immune response established in patients with spontaneous bacterial peritonitis. Hepatology 2008; 47 (3): 978-85.

30. Ahboucha S, Jiang W, Chatauret N, Mamer O, Baker GB, Butterworth RF. Indomethacin improves locomotor deficit and reduces brain concentrations of neuroinhibitory steroids in rats following portacaval anastomosis. Neurogastroenterol Motil 2008; 20 (8): 949-57.

31. Fisher CJ, Faloon WW. Blood ammonia levels in hepatic cirrhosis. Their control by the oral administration of neomycin. N Eng J Med 1957; 256: 1030-5.

32. Conn HO, Leevy CM, Vlahcevic ZR, et al. Comparison of lactulose and neomycin in the treatment of chronic portal-systemic encephalopathy. A double blind controlled trial. Gastroenterology 1977; 72 (4 Pt 1): 573-83.

33. Tarao K, Ikeda T, Hayashi K, et al. Successful use of vancomycin hydrochloride in the treatment of lactulose resistant chronic hepatic encephalopathy. Gut 1990; 31 (6): 702-6.

34. Rothenberg ME, Keeffe EB. Antibiotics in the management of hepatic encephalopathy: an evidence-based review. Rev Gastroenterol Disord 2005; 5 (Supl. 3): 26-35.

35. Curioso WH, Monkemuller KE. Neomycin should not be used to treat hepatic encephalopathy. BMJ 2001; 323 (7306): 233.

36. Forbes A, Murray-Lyon I. Vancomycin in resistant hepatic encephalopathy. Gut 1990; 31 (12): 1424.

37. Adachi JA, DuPont HL. Rifaximin: a novel nonabsorbed rifamycin for gastrointestinal disorders. Clin Infect Dis 2006; 42 (4): 541-7.

38. Mas A, Rodes J, Sunyer L, et al. Comparison of rifaximin and lactitol in the treatment of acute hepatic encephalopathy: results of a randomized, double-blind, double-dummy, controlled clinical trial. J Hepatol 2003; 38 (1): 51-8.

39. Cordoba J, Lopez-Hellin J, Planas M, et al. Normal protein diet for episodic hepatic encephalopathy: results of a randomized study. J Hepatol 2004; 41: 38-3.

40. Olde Damink SWM, Jalan R, Deutz NE, et al. The kidney plays a major role in the hyperammonemia seen after simulated or actual GI bleeding in patients with cirrhosis. Hepatology 2003; 37 (6): 127785 .

41. Kondrup J, Muller MJ. Energy and protein requirements of patients with chronic liver disease. J Hepatol 1997; 27 (1): 239-47.

42. Felipo V, Miñana D, Grisolia S. Control of urea synthesis and ammonia utilization in protein deprivation and refeeding. Arch Biochem Biophys 1991; 285: 351-6.

43. Plauth M, Cabre E, Riggio O, et al. ESPEN Guidelines on Enteral Nutrition: Liver disease. Clin Nutr 2006; 25 (2): 285-94.

44. Kondrup J, Nielsen K, Juul A. Effect of long-term refeeding on protein metabolism in patients with cirrhosis of the liver. Br J Nutr 1997; 77: 197-212.

45. Mullen KD, Weber FL, Jr. Role of nutrition in hepatic encephalopathy. Semin Liver Dis 1991; 11 (4): 292-304.

46. Marchesini G, Bianchi G, Merli M, et al. Nutritional supplementation with branched-chain amino acids in advanced cirrhosis: a doubleblind randomized trial. Gastroenterology 2003; 124: 1792-801.

47. Morgan MY. Branched chain amino acids in the management of chronic liver disease. Facts and fantasies. J Hepatol 1990; 11: 133-41.

48. Mullen KD, Jones EA. Natural benzodiazepines and hepatic encephalopathy. Semin Liver Dis 1996; 16 (3): 255-64.

49. Yurdaydin C, Walsh TJ, Engler HD, et al. Gut bacteria provide precursors of benzodiazepine receptor ligands in a rat model of hepatic encephalopathy. Brain Res 1995; 679 (1): 42-8.

50. Goulenok C, Bernard B, Cadranel JF, et al. Flumazenil vs. placebo in hepatic encephalopathy in patients with cirrhosis: a meta-analysis. Alimentary Pharmacology \& Therapeutics 2002; 16: 361-72.

51. Uflacker R, Silva A, d'Albuquerque LA, Piske RL, Mourao GS. Chronic portosystemic encephalopathy: embolization of portosystemic shunts. Radiology 1987; 165 (3): 721-5.

52. Cordoba J, Olive G, Alonso J, et al. Improvement of magnetic resonance spectroscopic abnormalities but not pallidal hyperintensity followed amelioration of hepatic encephalopathy after occlusion of a large spleno-renal shunt. J Hepatol 2001; 34: 176-8.

53. Riggio O, Masini A, Efrati C, et al. Pharmacological prophylaxis of hepatic encephalopathy after transjugular intrahepatic portosystemic shunt: a randomized controlled study. J Hepatol 2005; 42 (5): 674-9.

54. Maleux G, Heye S, Verslype C, Nevens F. Management of transjugular intrahepatic portosystemic shunt induced refractory hepatic encephalopathy with the parallel technique: results of a clinical followup study. J Vasc Interv Radiol 2007; 18 (8): 986-92.

55. Enns GM, Berry SA, Berry GT, Rhead WJ, Brusilow SW, Hamosh A. Survival after treatment with phenylacetate and benzoate for urea- 
cycle disorders. N Engl J Med 2007; 356 (22): 2282-92.

56. Kircheis G, Nilius R, Held C et al. Therapeutic efficacy of L-ornithine-L-aspartate infusions in patients with cirrhosis and hepatic encephalopathy: results of a placebo-controlled, double-blind study. Hepatology 1997; 25 (6): 1351-60.

57. Poo JL, Gongora J, Sanchez-Avila F, et al. Efficacy of oral L-ornithine-L-aspartate in cirrhotic patients with hyperammonemic hepatic encephalopathy. Results of a randomized, lactulose-controlled study. Ann Hepatol 2006; 5 (4): 281-8.

58. Jalan R, Wright G, Davies NA, Hodges SJ. 1-Ornithine phenylacetate (OP): A novel treatment for hyperammonemia and hepatic encepha- lopathy. Med Hypotheses 2007.

59. Liu Q, Duan ZP, Ha DK, Bengmark S, Kurtovic J, Riordan SM. Synbiotic modulation of gut flora: effect on minimal hepatic encephalopathy in patients with cirrhosis. Hepatology 2004; 39 (5): 1441-9.

60. Read AE, McCarthy CF, Heaton KW, Laidlaw J. Lactobacillus acidophilus (enpac) in treatment of hepatic encephalopathy. Br Med J 1966; 5498: 1267-9.

61. Hassanein TI, Tofteng F, Brown RS, Jr., et al. Randomized controlled study of extracorporeal albumin dialysis for hepatic encephalopathy in advanced cirrhosis. Hepatology 2007; 46 (6): 1853-62. 